Bull. Mater. Sci., Vol. 3, Number 2, July 1981, pp gestę () Printed in India.

\title{
Some interesting phase transitions in solids*
}

\author{
C N R RAO \\ Solid State and Structural Chemistry Unit, Indian Institute of Science, \\ Bangalore 560012 , India
}

MS received 18 January 1981

\begin{abstract}
A few types of phase transitions in solids of interest to the author are discussed after a brief presentation of the general features of phase transitions. The different systems discussed include low-dimensional solids, polytypes, organic solids, plastic crystals, glasses and silver selenide. Spin-state transitions and dipole glasses are also briefly examined.
\end{abstract}

Keywords. Phase transitions; plastic crystals; low-dimensional solids; spin-state transitions; glass transition; dipole glass; polytypism.

\section{Introduction}

Many solids undergo transformations from one crystal structure to another as the temperature or pressure is varied. While phase transitions generally involve changes in the atomic configurations in crystals, there are also transformations where electronic or spin configurations undergo changes. The subject of phase transitions is not only of great academic interest but also of technological importance. Phase transitions are exhibited by a wide variety of systems from simple metals and alloys to complex inorganic and organic materials (Rao and Rao 1978). In recent years, the subject has grown enormously with new types of transitions as well as new approaches to explain the phenomena being reported all the time. The importance of the subject can be judged by the prominent place it finds in a review of the progress of physics in recent decades (Bromley 1980); the subject also finds a place in a similar review of the frontiers of chemistry (Joyce 1980). In this presentation, a brief review of some general features of phase transitions is presented and results of some specific types of phase transitions of interest to us in this laboratory are discussed.

\section{Thermodynamics of phase transitions}

During a phase transition, the free energy of the solid remains continuous, but thermodynamic quantities like entropy, volume and heat capacity undergo discontinuous changes. Depending on which derivative of the Gibbs free energy shows

* Communication No. 109 from the Solid State \& Structural Chemistry Unit. Presented at the Biswas memorial symposium, December 1980. 

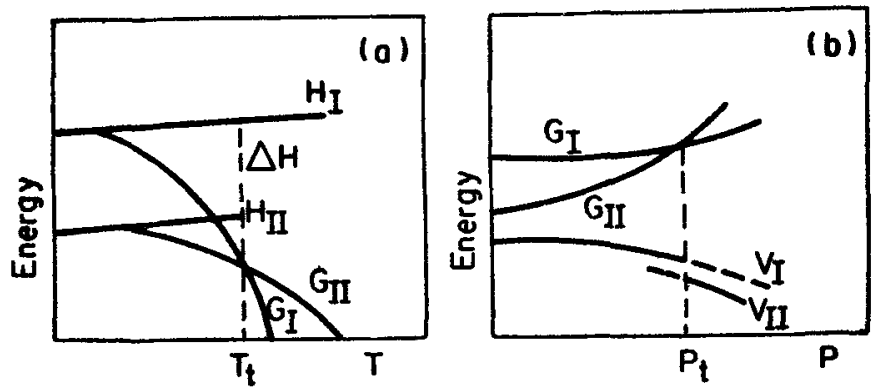

Figure 1. Variation of free energy and enthalpy with temperature and of free energy and volume with pressure in first order transitions.

a discontinuous change at the transition, phase transitions are classified as first order, second order and so on. A first order transition involving discontinuous changes in entropy and volume are shown in figure 1 . In this figure curves $G_{I}, G_{I I}$ represent variations in free energies of phases $I$ and II respectively while $H_{I}, H_{I I}$ and $V_{I}, V_{I I}$ represent variations in enthalpy and volume. We see that the free energy curves intersect $(\Delta \mathrm{G}=0)$ at the transition temperature $T_{t}$ or the transition pressure $P_{t}$.

In a second order transition, the $G(P, T)$ curves do not intersect sharply and we see a continuous variation in enthalpy or volume through the transition. We generally see changes in heat capacity in such transitions. Second order transitions are generally associated with some sort of disordering process. The long range order parameter $\xi$ varies with temperature in a second order transition, having a maximum value of 1 when there is perfect order and a value of zero when there is perfect disorder. There are a number of transitions where the heat capacity tends towards infinity at the transition temperature (giving rise to a lambda shaped $C_{P}$-temperature curve) and such transitions are referred to as $\lambda$-transitions.

The Clausius-Clapeyron equation, (1), describes the thermodynamics at a first order transition. In the case of second order transitions $\Delta V$ and $\Delta S$ have zero values and equation (1) will have a $0 / 0$ indeterminacy. We can, however, derive analogies of the Clausius-Clapeyron equation for these transitions as in equation (2)

$$
\begin{aligned}
& \frac{d p}{d T}=\frac{\Delta S}{\Delta V}=\frac{\Delta H}{T \Delta V}, \\
& \frac{d p}{d T}=\frac{\Delta C_{p}}{V T \Delta \alpha} .
\end{aligned}
$$

One of the most important developments in understanding the thermodynamics of structural phase transitions of second order is that duc to Landau who considered the free energy density $\phi$ to be a function of the order parameter as follows:

$$
\phi(P, T, \xi)=\phi_{0}(P, T)+a \xi+b \xi^{2}+c \xi^{3}+d \xi^{4}+\ldots
$$

where $\phi_{0}(P, T), a, b, c$ and $d$ are constants. Here, $\xi=1$ in the completely ordered phase (at low temperatures) and $\xi=0$ in the completely disordered phase after the 
transition. Since, changing the sign of $\xi$ should not alter the value of $\phi$, the coefficients of odd powers of $\xi$ should be equal to zero. Equation (3) then becomes,

$$
\phi(P, T, \xi)=\phi_{0}(P, T)+b \xi^{2}+d \xi^{4}+\ldots
$$

The equilibrium value of the long-range order parameter, $\xi$, is obtained by the conditions,

$$
\begin{aligned}
& \left(\frac{\partial \phi}{\partial \xi}\right)_{P, T}=\xi\left(b+2 d \xi^{2}\right)=0, \\
& \left(\frac{\partial^{2} \phi}{\partial \xi^{2}}\right)_{P, T}=\left(b+6 d \xi^{2}\right)>0 .
\end{aligned}
$$

From these relations we obtain solutions $\xi=0$ and $\xi^{2}=-b / 2 d$. Since $\xi=0$ in the disordered state, $b>0$ on one side of the transition temperature; we then find that $b<0$ for the ordered phase. That is, $b$ changes sign through a second order transition. Assuming $b$ to vary linearly with temperature, $b(P, T)=B\left(T-T_{c}\right)$ where $T_{c}$ is the transition (critical) temperature, we obtain

$$
\xi^{2}=-b / 2 d=-B\left(T-T_{c}\right) / 2 d
$$

It can be readily shown that the change in heat capacity at the transition is given by, $\Delta C_{p}=B^{2} T_{c} / 2 d$.

We thus see that Landau's theory provides the basis for second order transitions. The most important point to note is that the average value of the order parameter $\langle\xi\rangle$ vanishes above $T_{c}$ in a second order transition and is non-vanishing below $T_{c}$; in a first order transition, the change in $\langle\xi\rangle$ is discontinuous (figure 2). The identification of the order parameter is easy for some transitions and not as straight-

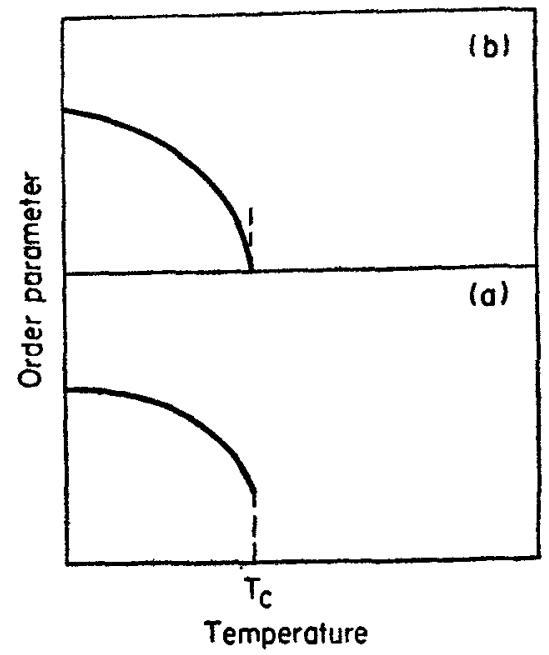

Figure 2. Variation of the order parameter with temperature (a) in a first-order transition and (b) in a second-order transition. 
forward in others. For example, in iron, the order parameter associated with the ferromagnetic-paramagnetic transition is the magnetisation.

Another important contribution of Landau is related to symmetry changes accompanying phase transitions. In second order transitions the symmetry of the crystal changes discontinuously causing the appearance (or disappearance) of certain symmetry elements, unlike first order transitions where there is no relation between the symmetries of the high and low temperature phases. If $\rho(x, y, z)$ describes the probability distribution of atom positions in a crystal, then $\rho$ would reflect the symmetry group of the crystal. This means, that for $T>T_{c}, \rho$ must be consistent with the symmetry group $G$ of the high temperature (high symmetry) phase. When $T<T_{c}, \rho$ must be consistent with the symmetry groups $G_{0}$ of the low-temperature (low symmetry) phase. We can write,

$$
\rho=\rho_{0}+\delta \rho=\rho_{0}+\Sigma C_{i} \psi_{i}^{\lambda}\left(\Gamma_{\lambda}\right)
$$

where $\psi_{i}^{\lambda}$ are basis functions transforming according to the irreducible representation $\Gamma_{\lambda}$ and $G_{0} ; \rho_{0}$ corresponds to the symmetry of $G_{0}$ and $\rho$ to the symmetry of $G ; \delta \rho$ has the same symmetry as $\rho$. Equation (4) is actually written as

$$
\phi=\phi_{0}(P, T)+b \xi^{2}+\xi^{4} \sum_{a} V_{a} f_{a}^{4}\left(\gamma_{i}\right)
$$

where $\xi^{2}=\Sigma_{i} C_{i}^{2}, C_{i}=\gamma_{i} \xi$ and $\Sigma_{i} \gamma_{i}^{2}=1$. The quantity $f_{a}^{4}$ is an invariant of fourth order constructed from $\psi_{i}$. We thus see that a transition from high symmetry $G$ to low symmetry $G_{0}$ accompanied by the appearance of an order parameter $\langle\xi\rangle$ occurs when $b$ changes sign.

\section{Soft modes}

As early as 1940 , Raman and Nedungadi discovered that the $\alpha-\beta$ transition of quartz was accompanied by a decrease in the frequency of a totally symmetric optic mode as the temperature approached the phase transition temperature. Historically, this is the first observation of a soft mode. Operationally, a soft mode is a collective excitation whose frequency decreases anomalously as the transition point is reached. In figure 3, we show the temperature dependence of soft mode frequencies. While in a second order transition, the soft mode frequency goes to zero at $T_{c}$, in a first order transition, the change of phase occurs before the mode frequency is able to go to zero. From figure 3, we see that soft modes exist both below and above $T_{c}$.

Prediction of soft modes was made independently by both Cochran and Anderson in 1959 who pointed out that phase transitions in some ferroelectrics may result from lattice dynamical instability. Indeed, Born and Huang (1954) had earlier shown that a crystal lattice becomes unstable if one of its normal mode frequencies becomes purely imaginary. Cowley in 1962 discovered from neutron scattering studies that one of the optic modes of $\mathrm{SrTiO}_{3}$ exhibited softening behaviour. In recent years soft modes have been found in a variety of systems including superconductors and organic solids (Blinc and Zeks 1974; Scott 1974; Rao and Rao 1978); the subject has been reviewed recently by Venkataraman (1979) in this Journal. We can understand the relation between soft modes and order parameters in phase transitions as follows. 


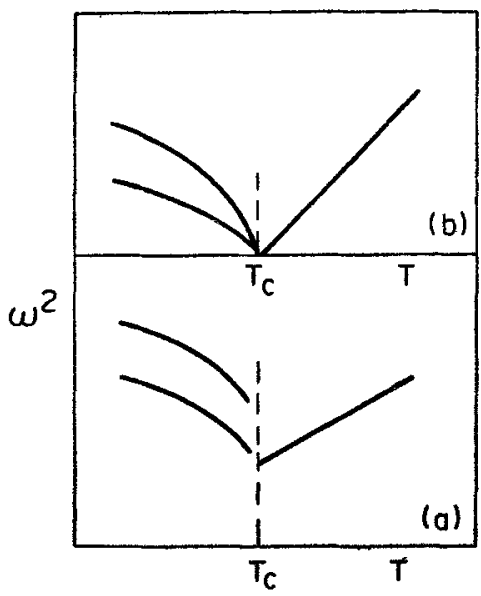

Figure 3. Variation of the square of the soft mode frequency, $\omega^{2}$ with temperature (a) in a first order transition and (b) in a second order transition.
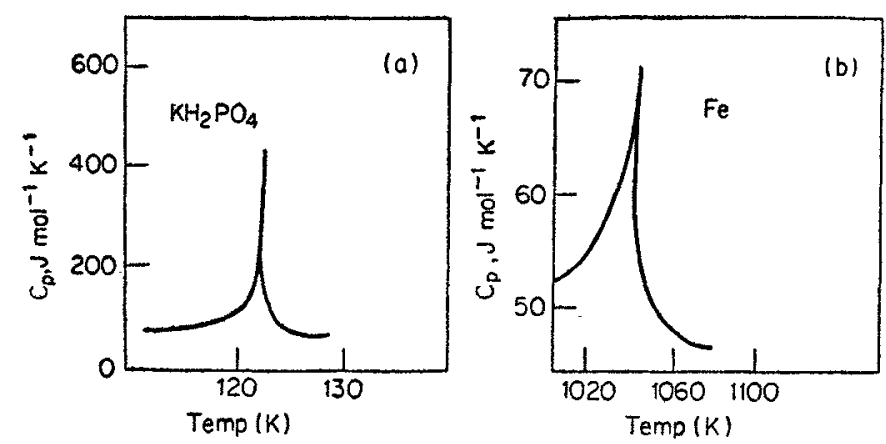

Figure 4. Heat capacity curves showing divergence at $T_{c}$ : (a) $\mathrm{KH}_{2} \mathrm{PO}_{4}$ and (b) $\mathrm{Fe}$.

The order parameter $\xi$ is not a static quantity although $\langle\xi\rangle$ is; $\xi$ can fluctuate and these fluctuations can be derivcd by a suitable superposition of the atomic displacements associated with soft mode vibrations. Landau's theory has been used widely to determine soft mode eigen vectors from experiments.

\section{Critical phenomena}

Many physical properties diverge near $T_{c}$ (i.e., show large values as $T_{c}$ is approached from either side). Interestingly, divergences of similar quantities in different phase transitions are strikingly similar as shown by typical $C_{p}$-temperature curves in figure 4. These divergences can be quantified in terms of the so-called critical exponents. A critical point exponent is given by,

$$
\lambda=\lim _{\epsilon \rightarrow 0}\left|\frac{\ln f(\epsilon)}{\ln |\epsilon|}\right|,
$$

where $\epsilon=\left(T-T_{c}\right) / T_{c}$ and $f(\epsilon)$ is the function whose exponent is $\lambda$. The most important exponents are those associated with the specific heat $(\alpha)$, the order para- 
meter $(\beta)$, the susceptibility $(\gamma)$ and the correlation length $(\nu)$. The correlation length refers to the range over which individual constituents (like atoms, atomic moments, etc.) are correlated. It so happens that the individual exponents for different transitions are roughly similar (e.g., $\beta \approx 0 \cdot 33$ ). More interesting is the fact that $\alpha+2 \beta+\gamma=2$ in all transitions independent of the detailed nature of the systcm whether it is a binary liquid mixture or a magnet.

Such a universality in critical exponents had intrigued scicntists for a long time, but is now understood in the light of Kadanoff's concept of scale invariance (Kadanoff 1966) associated with fluctuations near $T_{c}$. Wilson and Fisher (1972) employed the so-called renormalization group method to calculate the exponents. It has bec: found that all phase transitions can be characterized in terms of the dimensionality of the system and the dimensionality of the order parameter, the dimensionality of the system being treated as a continuously varying parameter. A detailed discussion of critical phenomena and the renormalization group would be outside the scope of this presentation and excellent reviews are available on the subject (Ma 1976; Fleury 1981). As Bromley (1980) points out, what is most surprising and gratifying about the renormalization group is the recognition that the entire formalism appropriately interpreted, has direct applicability in general field theory. This is yet another illustration of the unity of physical science.

\section{Structural changes in phase transitions}

On the basis of our knowledge of crystal chemistry we can predict the nature of structural changes in the phase transitions of simple ionic solids. In making such predictions we take advantage of the fact that in thermal transitions, the high-temperature form has higher symmetry than the low-temperature form. In pressure transitions, the high-pressure form will be more closely packed (higher coordination number) and will have a smaller volume. Thus, on application of pressure, $\mathrm{NaCl}$ type solids would give rise to $\mathrm{CsCl}$ type solids. Heating $\mathrm{CsCl}$ type solids, on the other hand, would give rise to $\mathrm{NaCl}$ type solids. In $\mathrm{AB}_{2}$ type solids, we could expect a distorted rutile or some other low symmetry structure to transform to the rutile or the fluorite structure at high temperature. A distorted perovskite would be expected to transform to the cubic structure at high temperatures.

Buerger (1951) classified phase transitions on the basis of structural changes involving primary or higher coordination as follows:

(i) Transformations involving first coordination (e.g. $\mathrm{CsC1}-\mathrm{NaCl}$, aragonitecalcite): (a) reconstructive (sluggish) (b) dilatational (rapid),

(ii) Transformations involving second or higher coordination (e.g. $\mathrm{BaTiO}_{3}$ : $\alpha-\beta$ quartz): (a) reconstructive (sluggish) (b) displacive (rapid).

While reconstructive transitions involving breaking and making of bonds would require high energy, the same can be accomplished by a simple dilatational mechanism. Buerger proposed such a mechanism for the transformation from $\mathrm{CsCl}$ structure to $\mathrm{NaCl}$ structure. Such deformational relations are known to exist between different structures (Rao and Rao 1978) and they are important in understanding mechanisms of phase transitions. In displacive transitions only small changes in the arrangement of coordination polyhedra occur. 


\section{Mechanisms of phase transitions}

Phase transitions in solids are also fruitfully classified on the basis of the mechanism. The important kinds of transitions normally encountered are: (i) nucleation and growth transitions; (ii) order-disorder transitions and (iii) martensitic transitions.

In nucleation-growth transitions, critical-sized nuclei of the new phase are first formed in the parent phase. The rate of nucleation can be shown to be given by,

$$
R=A \exp \left[-\left(\Delta G_{c}+E_{a}\right) / R T\right]
$$

where $A$ is the pre-exponential factor, $E_{a}$, the activation energy for the jump of atoms across the nucleus-bulk interface and $\Delta G_{c}$ is the free energy change in the formation of critical-sized nuclei. Once nucleation has occurred, phase transitions proceed towards completion by the growth or propagation of the critical-sized nuclei. The growth step will also be associated with a free energy of activation.

Order-disorder transitions are known to occur in a variety of crystals and the subject has been reviewed cxcellently by Parsonage and Staveley (1979). Martensitic transitions which were originally discovered in steel, are now considered to provide the mechanism for transitions in a variety of inorganic (and possibly even organic) solids (Rao and Rao 1978). A martensitic transition is a structural change caused by atomic displacements (and not by diffusion) corresponding to a homogeneous deformation which gives rise to an invariant plane strain through which the parent and product phases are related by a substitutional lattice correspondence, an irrational habit plane and a precise orientational relationship. The systematic change in atomic positions over macroscopic distances has led to the metaphoric description of these transitions as "military" transformations. Martensitic transitions often occur with very high velocities (of the order of sound velocity). It is interesting that the $\mathrm{CsCl}-\mathrm{NaCl}$ transition for which Buerger proposed a dilatational mechanism is indeed found to be a martensitic transition with orientational relations betwcen the two phases.

\section{Low dimensional materials}

Ever since Little (1964) suggested the possibility of high temperature superconductivity in one-dimensional organic materials, there have been considerable efforts in the study of these materials. Synthesis of such systems has not been very successful hitherto and there are serious theoretical objections to the observation of superconductivity in them. These are due to (i) occurrence of Peierls distortion in oncdimensional conductors, (ii) localized nature of one-electron states and (iii) possibility of a Mott transition to an insulating state due coulombic repulsion. Quasione-dimensional systems are therefore being explored to achieve the objective and the subject has been recently reviewed in this journal by Subramanyam (1979). Although initial experiments on systems like TTF-TCNQ were not successful, possibilities with $\mathbf{A}_{3} \mathrm{~B}$ compounds of $\beta$-tungsten structure deserve to be cxplored. Polymeric $(\mathrm{SN})_{x}$ has been found to be superconducting with a $T_{c}$ of $0.26 \mathrm{~K}$. Jerome et al (1980) have recently found a superconducting transition in di-(tetramethyl tetraselenafulvalene) hexafluorophosphate at $0.9 \mathrm{~K}$ at a pressure of $12 \mathrm{~K}$ bar. 
Similar transitions under pressure are likely to occur in coordination compounds with metal chains (like KCP) as well.

We have been interested in the origin of polytypism which is considered to be onedimensional polymorphism. We have treated polytypes as analogues of a onedimensional spin-half Ising chain with competing short - and infinite range interactions and have carried out computer simulations. Employing a Hamiltonian similar to that of Theumann and Hoye (1971), it has been possible to generate ordered polytypes up to $12 \mathrm{R}$ and short stretches of long period polytypes up to $33 \mathrm{R}$ (Uppal et al 1980). In figure 5 is shown the display of the $12 \mathrm{R}$ polytype simulated by us. The studies have shown that it is indecd possible to understand polytypism based on thermodynamic considerations. Observation of long-period sequences in the computer simulation could be of significance in the context of Frank's theory of polytypism. We have carried out simulation studies under different conditions of growth by varying the nature of short-range interactions having justified the form of interactions itself by model potential calculations.

In the last few years we have been interested in the phase transitions of compounds of the type $\left(\mathrm{C}_{n} \mathrm{H}_{2 n+1} \mathrm{NH}_{3}\right)_{2} \mathrm{MCl}_{4}, \mathrm{M}=\mathrm{Mn}, \mathrm{Fe}, \mathrm{Cd}$ or $\mathrm{Cu}$, which provide interesting model systems to investigate magnetic phenomena in two dimensions. The transition metal ions can interact only in two dimensions because of the presence of the alkylammonium groups above and below the metal-chlorine clusters (figure 6). Magnetic resonance and other spectroscopic investigations (Kind and Roos 1976; Blinc et al 1976; Adams and Stevens 1978) seem to show that in $\left(\mathrm{CH}_{3} \mathrm{NH}_{3}\right)_{2} \mathrm{MCl}_{4}$, the phase transitions are determined essentially by the motions of the methylammonium groups, the metal-chlorine clusters affecting the phase transitions only indirectly (Blinc et al 1978). We have examined the infrared spectra of several $\left(\mathrm{C}_{n} \mathrm{H}_{2 n+1} \mathrm{NH}_{3}\right)_{2} \mathrm{MCl}_{4}$

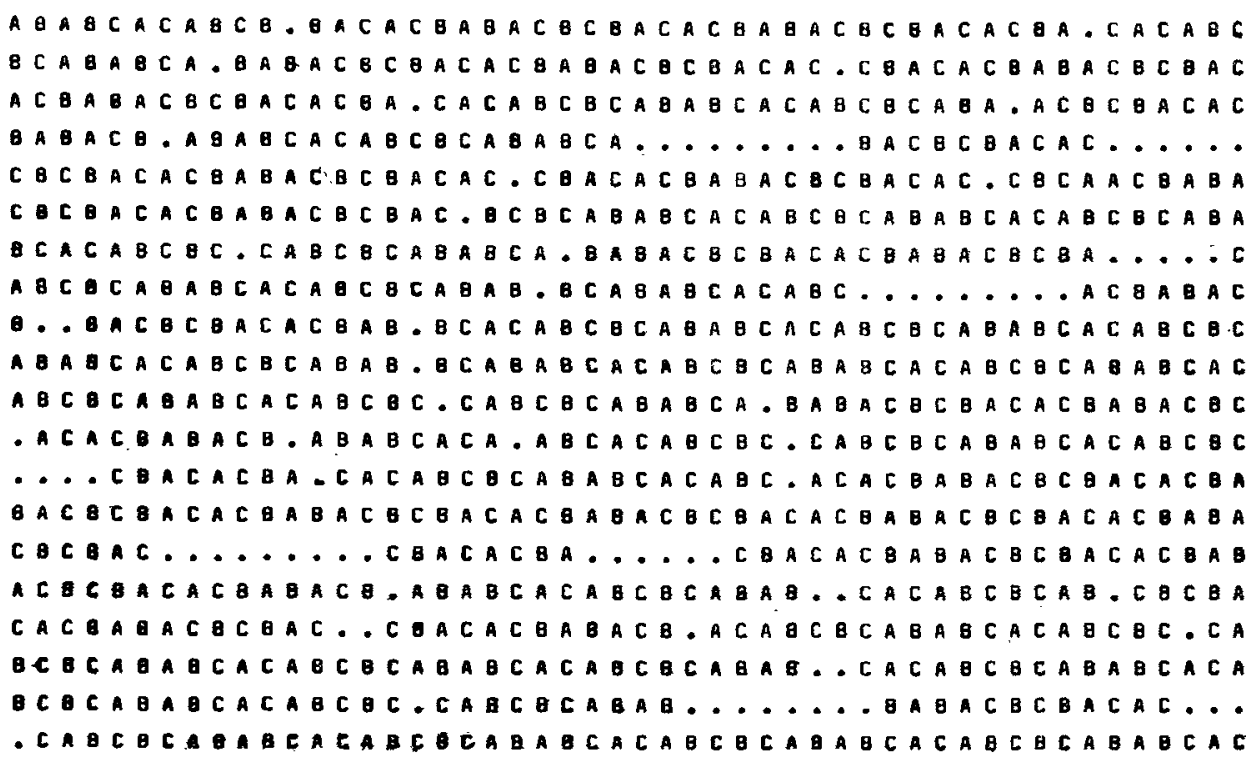

Figure 5. Computer display of $12 \mathrm{R}$ polytype. 


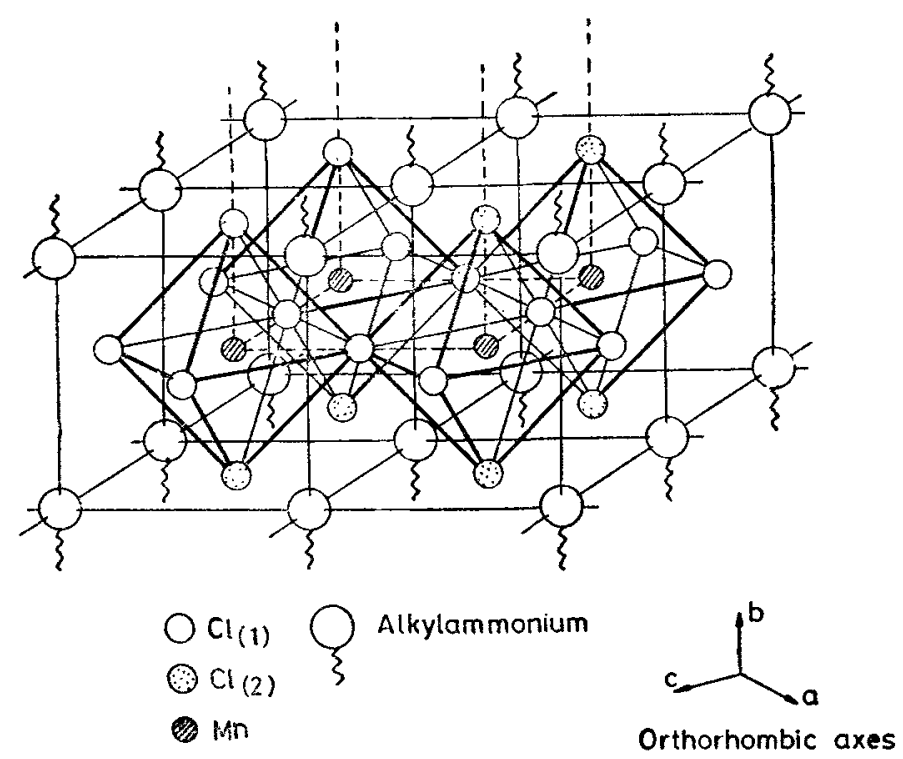

Figure 6. Structure of $\left(\mathrm{CH}_{3} \mathrm{NH}_{3}\right)_{2} \mathrm{MnCl}_{4}$

systems through their phase transitions to investigate whether the intramolecular vibration modes show the expected changes. In the high-temperature phases of these solids the $\left(\mathrm{CH}_{3} \mathrm{NH}_{3}\right)^{+}$ion will have $\mathrm{C}_{3 v}$ symmetry but the symmetry goes down to $C_{1}$ or $C_{s}$ in the ordered low-temperature phases. The infrared spectra do indeed show the expected site-group as well as factor-group splittings in the lowtemperature phases, the degenerate bending modes of $\mathrm{NH}_{3}$ and $\mathrm{CH}_{3}$ being particularly sensitive (figure 7). Our studies clearly show that the phase transitions of the tetrachlorometallate are similar to those of the corresponding alkylammonium chlorides, $\mathrm{C}_{n} \mathrm{H}_{2 n+1} \mathrm{NH}_{3} \mathrm{Cl}$, thereby establishing that the phase transitions in the former are entirely controlled by the motions of the $\left(\mathrm{C}_{n} \mathrm{H}_{2 n+1} \mathrm{NH}_{3}\right)^{+}$group. Accordingly, we find $\left(\mathrm{C}_{n} \mathrm{H}_{2 n+1} \mathrm{NH}_{3}\right)_{2} \mathrm{MBr}_{4}$ systems also show transitions similar to those of the chloro compounds, just as $\mathrm{C}_{n} \mathrm{H}_{2 n+1} \mathrm{NH}_{3} \mathrm{Br}$ show transitions akin to the corresponding chlorides.

\section{Organic solids}

Although phase transitions in organic solids have been reported from time to time, extensive mechanistic studies have not been carried out as in the case of inorganic solids. It was believed until recently by many workers that there are no structural relations between phases in the case of organic solids. Jones et al (1975) have reported that the stress-induced phase transition of 1,8-dichloro-10-methylanthracene proceeds by a diffusionless displacive transition (somewhat similar to a martensitic transition) with definite orientational relationships. The irrational habit plane seems to be composed of close packed planes and the properties of the interface could be formulated in terms of dislocations. The phase transition of 5-methyl-1-thia-5-azoniacyclo-octane-1-oxide perchlorate has been explained by Parkinson et al (1976) in terms of recurrent glissile partial dislocations; it was 


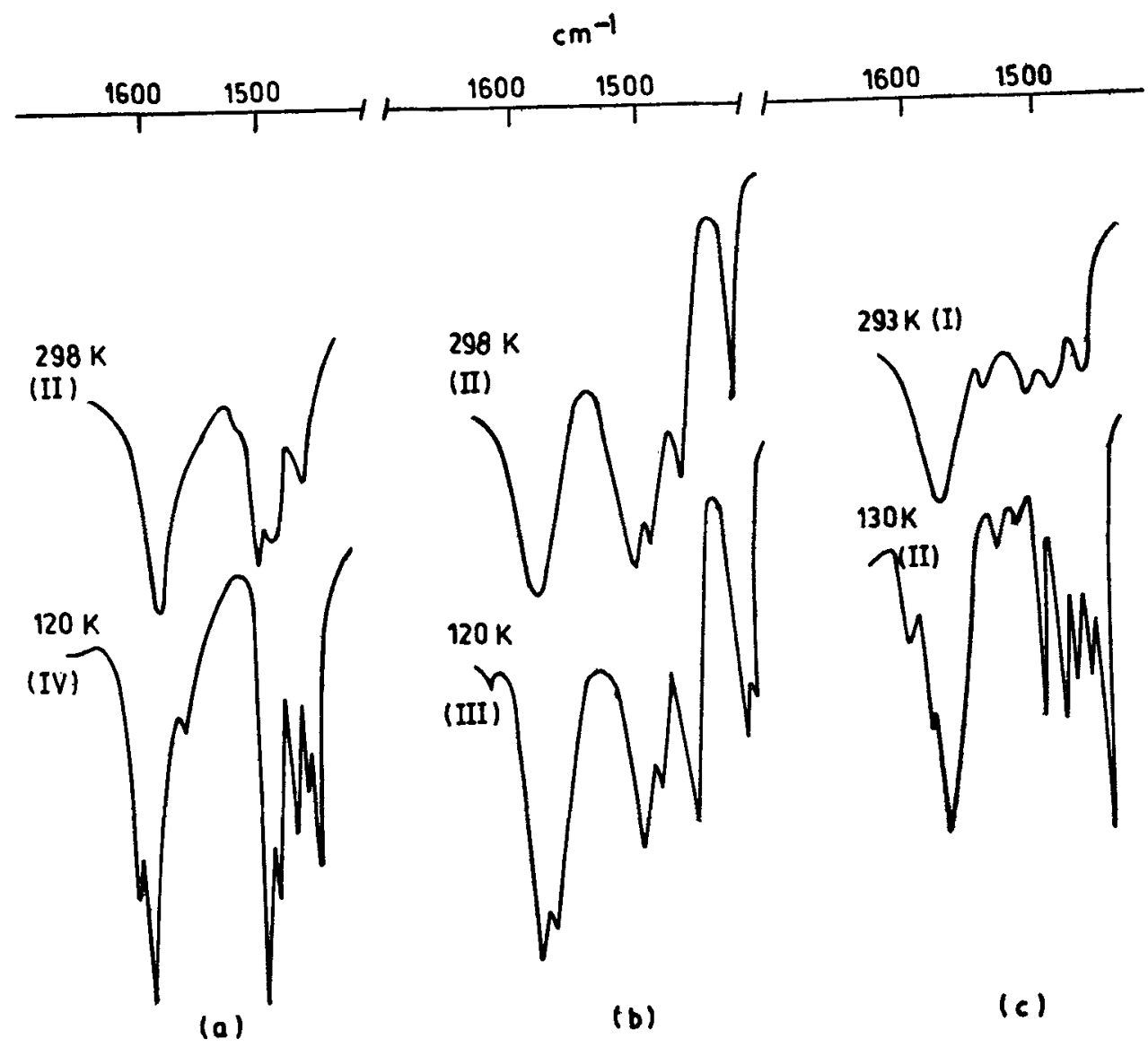

Figure 7. Infrared spectra of $\left(\mathrm{CH}_{3} \mathrm{NH}_{3}\right)_{2} \mathrm{MCl}_{4}$ systems in the $1600-1450 \mathrm{~cm}^{-1}$ region. Note the change in the asymmetric $\mathrm{NH}_{3}$ and $\mathrm{CH}_{3}$ deformation bands around $1590 \mathrm{~cm}^{-1}$ and $1460 \mathrm{~cm}^{1}$ respectively: (a) $\mathrm{Cd}$, (b) $\mathrm{Mn}$ and (c) $\mathrm{Cu}$.

earlier thought that the transition involved a cooperative process of inversion and rotation of half of the molecular cations. In this laboratory, we have been studying phase transitions of a variety of organic solids. For example, we have investigated the $\alpha-\gamma-\alpha-\beta$ transitions of $p$-dichlorobenzene by infrared spectroscopy. The $\gamma$ phase is characterized by unusually high intramolecular vibration mode frequencies. The $\alpha-\gamma$ transition shows athermal nucleation behaviour as in martensitic transitions; the $\alpha-\beta$ transition seems to be associated with some orientational disorder (Ganguly et al 1979). We have found order-disorder transitions in benzothiophene and cyclopentyl methyl sulphide to be accompanied by marked variations of band intensities in infrared spectra. The electronic transition of KTCNQ is accompanied by marked changes in the infrared spectrum reflecting dimerization in the lowtemperature phase. Infrared spectroscopy has been employed by us to examine phase transitions of dicarboxylic acids. Thus, the transition of malonic acid around $355 \mathrm{~K}$ is accompanied by interesting changes in the hydrogen bond structure as revealed by infrared spectroscopy (Ganguly et al 1980). 
The study of the plastic crystalline state of organic compounds has become very popular recently (Sherwood 1979). We have examined the plastic state of several organic compounds by colorimetry as well as by infrared and ESR spectroscopy. The plastic state is characterised by a high value of $\Delta S$ of formation from the crystal state, the $\Delta S$ of the plastic-liquid transition being much smaller. Our studies show that the $\Delta H$ (as well as $\Delta S$ ) of the crystal-plastic transition generally decreases as the temperature range of stability of the plastic phase $\left(\Delta T_{p l}=T_{p l}-T_{c p}\right.$ where $T$ represents the transition temperature) increases. The $\Delta H$ (as well as the $\Delta S$ ) of the plastic-liquid transition, on the other hand, increases as the $\Delta T_{p l}$ increases. These trends are shown in figure 8 . We have examined correlation functions of

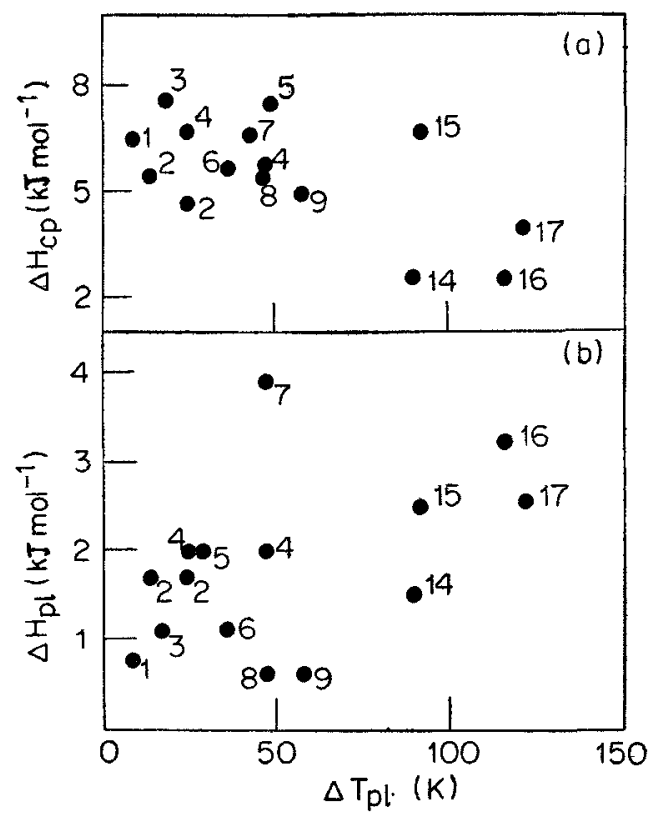

Figure 8. Variation of $\Delta H$ of (a) crystal-plastic and (b) plastic-liquid transitions with the plastic-liquid range, $\Delta T_{p l}$.

molecules in the plastic state by employing infrared band shapes. The correlation functions represent dipole rotational functions to an appreciable extent although some contribution from vibrational relaxation is undoubtedly present. The correlation times are continuous through the plastic-liquid transition (figure 9) and parallel the variation of viscosity. The tumbling correlation times of a spin-probe are also found to be continuous through the plastic-liquid transition. Activation energies for molecular motion seem to vary in the same direction as the $\Delta H$ of the plasticliquid transition. Infrared correlation times of solute molecules in binary mixtures in the plastic and the liquid states show interesting changes with solute concentration.

\section{Glasses}

One of the primary questions related to the glassy state that is not completely answered as yet is regarding the nature of the glass transition (Rao 1979). The 


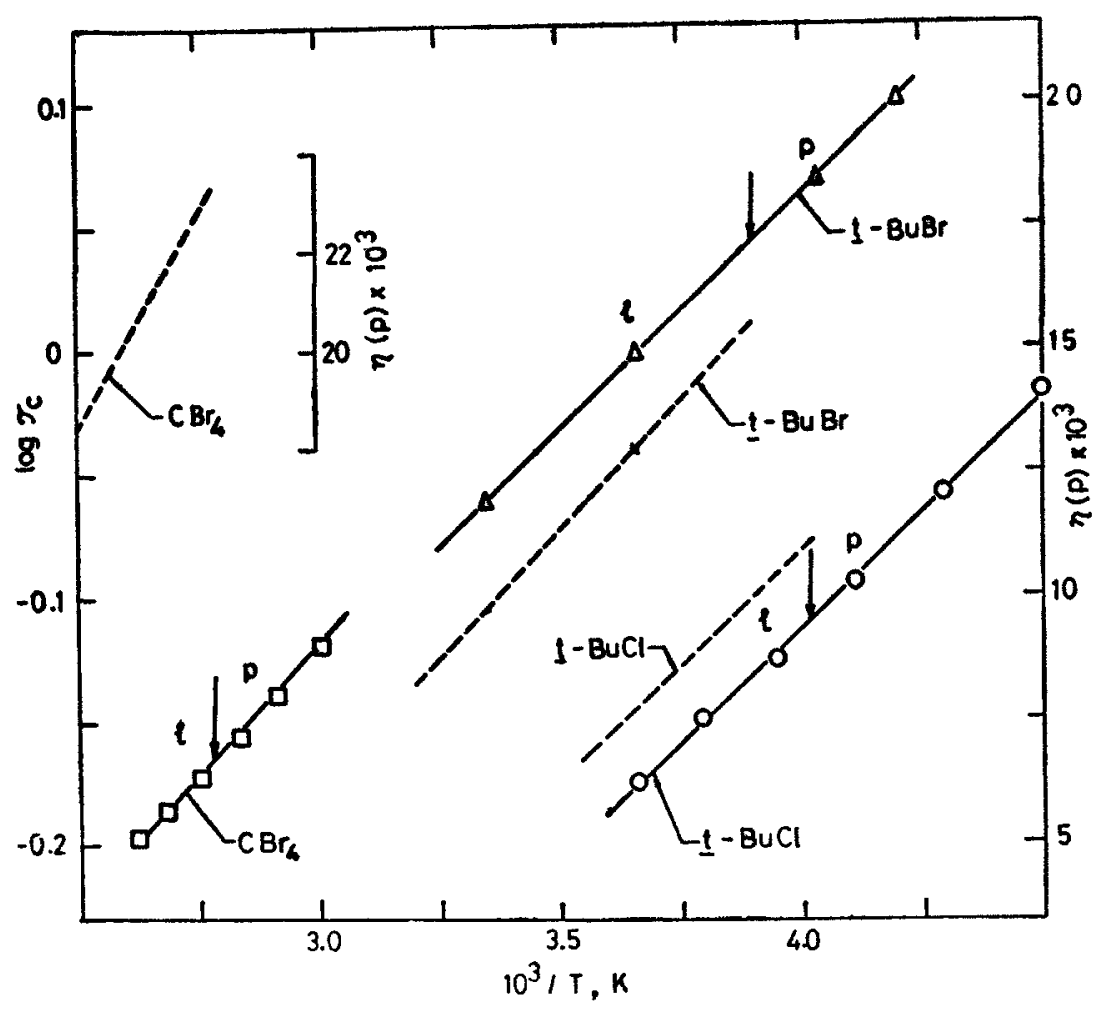

Figure 9. Plots of the logarithm of the infrared correlation times with reciprocal of temperature. Arrows show the plastic-liquid transition. Broken curves show plots of viscosity, $\eta$, against $1 / T . \quad \tau_{c}$ is in picoseconds.

value of the glass transition temperature $T_{g}$ itself is dependent on the rate of cooling. Yet $T_{g}$ scales as the melting or liquidus temperature suggesting a thermodynamic origin. Many properties of glasses, $P$, such as reciprocal of viscosity or relaxation time, diffusion coefficient, ionic conductivity and so on can be described by the relation,

$$
P=\text { constant } \times \exp \left[-E_{a} /\left(T-T_{0}\right)\right]
$$

Many have considered $T_{0}$ to be a thermodynamic transition temperature. Recently, Venkataraman (1980) has discussed the role of defects and suggests a KosterlitzThouless (1973) kind of change occurring at $T_{0}$. What is still intriguing is that many measurements on glasses follow a simple Arrhenius behaviour. It appears that the nature of $T_{g}$ and $T_{0}$ are still far from being completely understood.

The area of spin glasses has been very active in the past few years. In this laboratory we have been carrying out Monte-Carlo simulations of spin glasses and micto magnets. In the case of micto magnets we have found the cluster size to increase with diffusion. Heat capacity and magnetic susceptibility of these systems are nicely predicted in our simulation studies. What has been of greater interest to us recently is the possible occurrence of dipole glasses. Amongst several approaches to the study of dipole glasses, mention must be made of the dielectric properties of dilute 
solutions of ferroelectrics (like $\mathrm{PbTiO}_{3}$ or $\mathrm{KNbO}_{3}$ ) in antiferroelectrics (like $\mathrm{PbZrO}_{3}$ or $\mathrm{NaNbO}_{3}$ ). We have reason to believe that dipole clusters are formed in the solute composition range of $1-5$ mole $\%$ in these systems. It is noteworthy that Kirkpatrick and Verma (1976) have proposed a dipolar glass based on a random Ising model with $1 / R^{3}$ interactions; anomalous $T^{3 / 2}$ specific heat in off-centre ion doped alkali halides has been explained on the basis of this model. Hochli et al (1979) propose a polar glass in $\mathrm{KTaO}_{3}: \mathrm{Li}$ system based on relaxation and electret polarization effects.

\section{Electronic transitions}

The field of metal-insulator transitions has been actively explored for the past several years (Mott 1974) and we shall not discuss them in this presentation. One of the unusual types of electron transitions is that found in $\mathrm{LaCoO}_{3}$ which shows a first order phase transition around $1200 \mathrm{~K}$ without any change in crystal symmetry (Raccah and Goodenough 1967). The transition has been attributed to the change in the nature of $d$-electrons from localized behaviour to itincrant behaviour. We have been investigating these electronic transitions in several rare earth cobaltates of the general formula, $\mathrm{LnCoO}_{3}$. We have employed $\mathrm{Mössbauer}$ spectroscopy and a variety of other techniques to study the transitions (see for example Jadhao et al 1976). The studies have enabled us not only to follow the nature of changes occurring in cobalt states, but also to underscore the role of the rare earth ion. We have examined the $\mathrm{LaCoO}_{3}^{\prime}$ transition by $\mathrm{x}$-ray photoelectron spectroscopy (Thornton et al 1976) to understand the nature of changes in the valence band as wcll as in cobalt core levels. We have also employed photoelectron spectroscopy to follow metalinsulator transitions (Vasudevan et al 1978). He I photoelectron spectra clearly show the appearance of a sharp Fermi edge as an insulator transforms to the metallic state (figure 10).

One of the most interesting electronic transitions that we have recently examined is that in the mixed conductor $\mathrm{Ag}_{2+\delta}$ Se. This material undergoes an $\alpha-\beta$ transition where in the high-temperature $\beta$ phase, the $\mathrm{Ag}^{+}$ions are randomly distributed (just as in AgI). The electronic conductivity of $\mathrm{Ag}_{2+\delta}$ Se shows an interesting transition as a function of stoichiometry (figure 11). The cilver-excess material is metallic in the $\beta$-phase, but the silver-deficient material exhibits an activated transport. Since $\mathrm{Ag}_{2} \mathrm{Se}$ has a small energy gap, one could, in principle, consider the transition in electronic conductivity at intermediate $\delta$ values to be one from a small energy gap regime to one of a still smaller energy gap regime. One could also invoke disorderbased arguments, but it may not be necessary.

\section{Spin-state transitions}

Spin-state transitions occur in molecular systems of transition metal complexes and in transition metal oxides. The transitions can be gradual or abrupt and are often accompanied by structural transitions. In some systems, the high-spin population is non-zero at cryogenic temperatures. In an attempt to explain the various features of these transitions we have recently explored several theoretical models (Ramasesha et al 1979). Recent magnetic susceptibility studies (Madhusudan et al 
1980) show that the behaviour of $\mathrm{LaCoO}_{3}$ is entirely different from those of other rare earth cobaltates. While $\mathrm{LaCoO}_{3}$ shows a plateau in the inverse susceptibilitytemperature curve, the other cobaltates show a maximum. These two behaviours
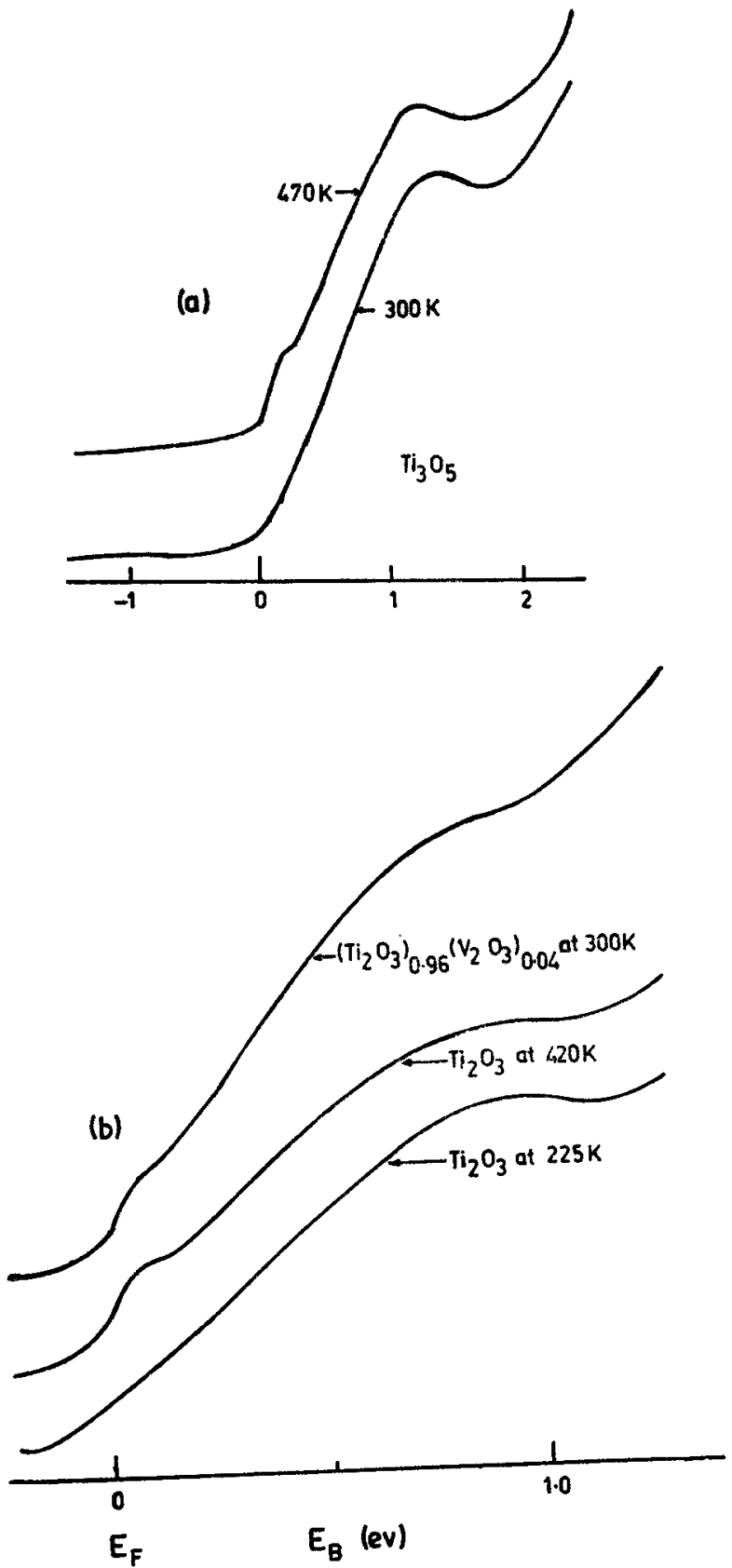

Figure 10. (a) He I valence band of $\mathrm{Ti}_{3} \mathrm{O}_{5}$ above and below the insulator-metal transition temperature. (b) He I valence band of $\mathrm{Ti}_{2} \mathrm{O}_{3}$ above and below the insulator. metal transition temperature; the band of $\left(\mathrm{Ti}_{2} \mathrm{O}_{3}\right)_{0.96}\left(\mathrm{~V}_{2} \mathrm{O}_{3}\right)_{0.04}$ which is metallic at room temperature is shown for comparison. 


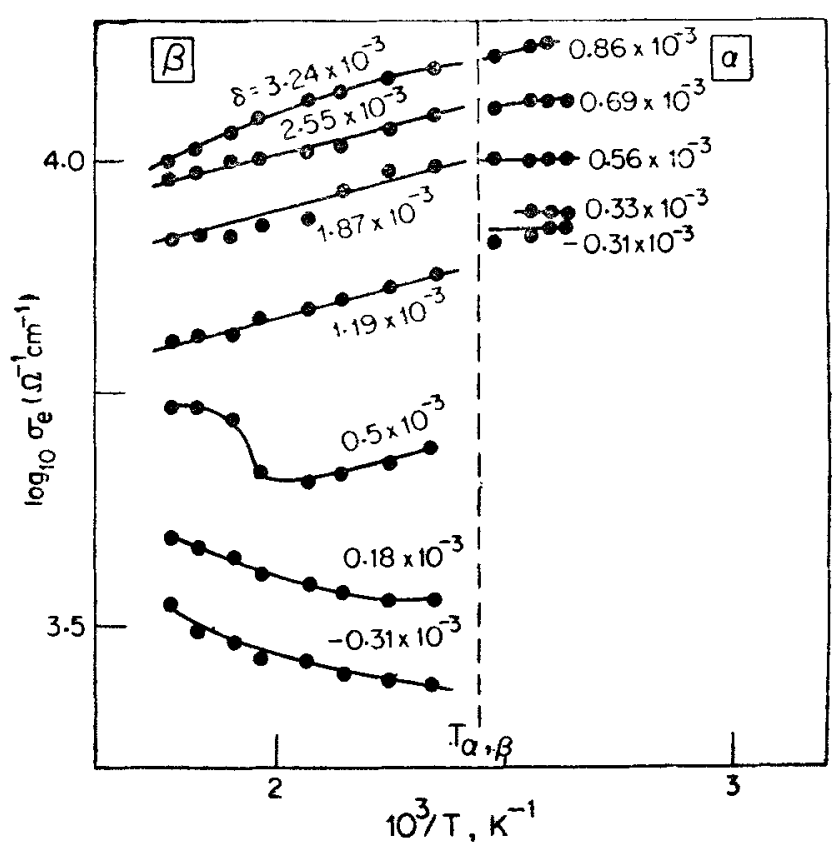

Figure 11. Variation of electrical conductivity of $\mathrm{Ag}_{2+\delta}$ Se with temperature (for different values of $\delta$ ) in $a$ and $\beta$ phases.

conform to different models of Ramasesha et al (1979). We have investigated spin state transitions in metal complexes and rare earth cobaltates by employing $\mathrm{x}$-ray photoelectron spectroscopy (Vasudevan et al 1979). Final state effects in the $d$-bands as well as satellites in the core levels are found useful in characterizing the high spin state. By cmploying ${ }^{59} \mathrm{Co} N \mathrm{NR}$, spin-state transitions in rare earth cobaltates have bcen examined by us recently (Bose et al 1980).

\section{Acknowledgement}

The author is thankful to Drs J $\mathrm{R}$ Fernandes, $\mathrm{P}$ Ganguly, $\mathrm{M} \mathrm{S}$ Hegde, S Ramasesha, K J Rao, A K Shukla, M K Uppal, S Vasudevan and Messrs S Ganguly, W H Madhusudan and H N Vasan for their collaboration in studies of phase transitions in this laboratory.

\section{References}

Adams D M and Stevens D C 1978 J. Phys. C11 617

Anderson P W 1959 in Fizika Dielektrikov (ed) G I Skanavi Akad Nauk SSR Moscow

Blinc R and Zeks B 1974 Soft modes in ferroelectrics and antiferroelectrics (Amsterdem : North Holland)

Blinc R, Burgar B, Lozar B, Seliger J, Slak J, Rutar V, Arend H and Kind R 1976 J. Chem. Phys. 66 278

Blinc R, Zeks B and Kind R 1978 Phys. Rev. 173409

Born M and Huang K 1954 Dynamical theory of crystal lattices (Oxford : Clarendon Press)

Bose M, Ghoshray A, Basu A and Rao C N R 1980 Chem. Phys. Lett. 74413 
Bromley D A 1980 Science 209110

Buerger M J 1951 in Phase transformations in solids (eds.) R Smoluchowski, J E Mayer and W A Weyl

(New York : John Wiley)

Cochran W 1959 Phys. Rev. Lett. 3412

Cowley R A 1962 Phys. Rev. Lett. 9195

Fleury P A 1981 Science 211125

Ganguly S, Fernandes J R, Bahadur D and Rao C N R 1979 J. Chem. Soc. Faraday Trans. 1175923

Ganguly S, Fernandes J R, Desiraju G and Rao C N R 1980 Chem. Phys. Lett. 69222

Hochli U T, Weibel H E and Boatner L A 1979 J. Phys. C12 L563

Jadhao V G, Singru R M, Bahadur D and Rao C N R 1976 J. Phys. Chem. Solids 37113

Jerome D, Mazaud A, Ribault M and Bechgaard K 1980 J. Phys. Lett. 41 L95

Jones W, Thomas J M and Williams J O 1975 Philos. Mag. 321

Joyce R M 1980 Science 20989

Kadanoff L P 1966 Physics 2263

Kind R and Roos J 1976 Phys. Rev. B13 45

Kirkpatrick S and Verma C M 1976 Solid State Commun. 25821

Kosterlitz J M and Thouless D J 1973 J. Phys C6 1181

Little W A 1964 Phys. Rev. 1341416

Ma S K 1976 Modern theory of critical phenomena (Reading Mass: Benjamin)

Madhusudan W H, Jagannathan K, Ganguly P and Rao C N R 1980 J.Chem. Soc. Dalton Trans.1397

Parkinson G M, Thomas J M, Williams J O, Goringe M J and Hobbs L W $1976 \mathrm{~J}$. Chem. Soc. Perkin II, 836

Parsonage N G and Staveley L A K 1979 Disorder in crystals (Oxford: University Press)

Raccah P M and Goodenough J M 1967 Phys. Rev. 155932

Raman C V and Nedungadi T M K 1940 Nature (London) 145147

Ramasesha S, Ramakrishnan T V and Rao C N R 1979 J. Phys. C12 1307

Rao C N R and Rao K J 1978 Phase transitions in solids (New York: McGraw Hill)

Rao K J 1979 Bull. Mater. Sci. 1181

Scott J F 1974 Rev. Mod. Phys. 4683

Sherwood J N 1979 The plastically crystalline state (London: John Wiley)

Subramanyam S V 1979 Bull. Mater. Sci. 1207

Theumann W K and Hoye J S 1971 J. Chem. Phys. 554159

Thornton G, Orchard A F and Rao C N R 1976 J. Phys. C9 1991

Uppal M K, Ramasesha S and Rao C N R 1980 Acta Crystallogr. A36 356

Vasudevan S, Hegde M S and Rao C N R 1978 Solid State Commun. 27131

Vasudevan S, Vasan H N and Rao C N R 1979 Chem. Phys. Lett. 65444

Venkataraman G 1979 Bull. Mater. Sci. 1129

Venkataraman G 1980 Lecture delivered at the Symposium on Metallic Glasses, Department of

Metallurgy, Banaras Hindu University, Varanasi (To be published in Bull. Mater. Sci.)

Wilson K G and Fisher M E 1972 Phys. Rev. Lett. 28240 